\title{
Time-of-Failure Probability Mass Function Computation Using the First-Passage-Time Method Applied to Particle Filter-based Prognostics
}

\author{
Francisco Jaramillo ${ }^{1}$, Martín Valderrama ${ }^{2}$, Vanessa Quintero ${ }^{3}$, Aramis Pérez $^{4}$, and Marcos Orchard ${ }^{5}$ \\ 1,2,5 Department of Electrical Engineering, University of Chile, Av. Tupper 2007, Santiago, Chile \\ francisco.jaramillo@ing.uchile.cl \\ martin.valderrama@ug.uchile.cl \\ morchard@ing.uchile.cl \\ ${ }^{3}$ Faculty of Electrical Engineering, Universidad Tecnológica de Panamá, C.R. Panamá Oeste, Panamá \\ vanessa.quintero1@utp.ac.pa \\ ${ }^{4}$ School of Electrical Engineering, University of Costa Rica, San Jose, Costa Rica \\ aramis.perez@ucr.ac.cr
}

\begin{abstract}
One of the main challenges in prognostics corresponds to the estimation of the probability density function (PDF) of the system's time-of-failure $(\mathrm{ToF})$ prior to reach a fault condition. An appropriate characterization of the ToF-PDF will let the user know about the remaining useful life of the system or component, allowing the users to prevent catastrophic failures through optimal maintenance schedules. However, the ToF-PDF estimation is not an easy task because it involves both the computation of long-term predictions of a fault indicator of the system and the definition of the hazard zone. In most cases, the trajectory of the fault indicator is assumed as a trajectory with monotonic behavior, and the hazard zone may be considered as a deterministic or probabilistic threshold. This monotonic behavior of the fault indicator enables assuming that the system will only fail once when this indicator reaches the hazard zone, and the ToF-PDF will be estimated according to mathematical definitions proposed in the state-of-the-art. Nevertheless, not all the fault indicators may be considered with a monotonic behavior due to its nature as a stochastic process or regeneration phenomenon, which may entail to errors in the ToF-PDF estimation. To overcome this issue, this paper presents an approach for the estimation of the ToF-PDF using the first-passage-time (FPT) method. This method is focused on the computation of the FPT-PDF when the stochastic process under analysis reaches a specified threshold for the first time only. Accordingly, this work aims

\footnotetext{
Francisco Jaramillo et al. This is an open-access article distributed under the terms of the Creative Commons Attribution 3.0 United States License, which permits unrestricted use, distribution, and reproduction in any medium, provided the original author and source are credited.
}

to analyze the impact in the estimation of the ToF-PMF (probability mass function) when particle-filter-based prognostics algorithms are used to perform long-term predictions of the fault indicator and compute the probability of failure considering specific hazard zones (which may be characterized by a deterministic value or by a failure likelihood function). A hypothetical self-regenerative degradation process is used as a case study to evaluate the performance of the proposed methods.

\section{INTRODUCTION}

Monitoring the state-of-health ( $\mathrm{SoH})$ of a system (and/or its components) is essential to improve their overall performance and reduce costs associated with a corrective maintenance (Wang, Lu, Cheng, \& Jiang, 2019). Based on this, prognostics and health management (PHM) plays a crucial role in the estimation of system conditions. According to $\mathrm{Si}$ (2015), PHM provides a set of tools used to guarantee the system's reliability, estimate its real condition, and avoid risks that can affect the operation or cause irreversible damage to the system. Prognostics allow the identification of the requirements of a system (and/or its components) in the future; that is, the time-of-failure (ToF) and the remaining useful life (RUL). The ToF is a relevant parameter in PHM, and it is defined as the time in which the failure threshold is reached (Skaf, 2015). In the case of RUL, it is defined as the difference between the ToF and the current time instant (Wei, Dong, \& Chen, 2018). In this article, we prefer to use ToF instead of RUL, since it is more general and is applicable for a broader range of cases (Orchard \& Vachtsevanos, 2009). 
Currently, various methods are used to estimate the ToF. Some authors have classified these methods into two major categories: model-based and data-driven. The first category consists of the use of a set of equations that include the physical characteristics of the phenomenon under study. In contrast, the second category uses a large amount of data obtained from sensors and monitoring. These data is then used to infer the behavior of the event. For example, Pola et al. (2015) presents a model-based approach combined with a particle filter (PF) to estimate the end of discharge (EoD) of lithium-ion batteries, while Liu, Zhao, and Peng (2019) presents a data-driven approach based on long short term memory networks and Bayesian model averaging to determine the RUL.

The SoH is an indicator widely used to characterize degradation processes. According to Qu, Liu, Ma, and Fan (2019), an accurate estimation of the SoH of a system will affect the RUL prediction directly. In this line, failure prognostic algorithms use long-term predictions to describe the future behavior of this kind of indicators to estimate the ToF of the faulty system. To achieve this, failure prognostic algorithms require a correct characterization of the indicator under study, and its accuracy has a direct impact on the estimation of the ToF probability mass function (ToF-PMF). An example of these algorithms is the one based on PFs, which are widely used by many researchers in diverse applications within the PHM community (Rozas et al., 2020).

The characterization of specific degradation processes continues to be a topic of interest in PHM for applications related to self-regenerative processes; such is the case of the discharging process for lithium-ion (Li-ion) batteries $(\mathrm{Ng}, \mathrm{Xing}$, \& Tsui, 2014; Zou, Hu, Ma, \& Li, 2015). According to Xu et al. (2019), the self-regenerative process in Li-ion batteries can be defined as the process by which the battery increases its capacity for the next operation cycle if a long standby time is considered. If this self-regenerative behavior is not treated adequately by prognostic algorithms, then the accuracy and precision of the ToF (or RUL) estimation may be considerably affected. Therefore, it is of utmost importance to characterize and consider aspects related to these processes through methodologies that allow computing a correct ToF (or RUL) estimation. Examples of advances in this area are the works presented in Orchard et al. (2015) and Xu et al. (2019).

This paper presents an approach to compute the ToF-PMF based on the concept of the first-passage-time (FPT) method. FPT is defined as the first time when a stochastic process crosses a specified threshold (Jaskowski \& van Dijk, 2015). This method is commonly used in the areas of economics and finance (Bakshi \& Panayotov, 2010; Janssen, Manca, \& Manca, 2013). However, during the last year, the concept was extended to different areas, such as animals' movement to establish a distant relationship between the animal and its prey (McKenzie, Lewis, \& Merrill, 2009). Another case is the study of a stochastic degradation model under bivariate time scales. In this specific study, the concept of FPT is used to predict the RUL of the degrading components (Pei et al., 2019). Finally, the approach proposed by Si (2015) combines the FPT concept with the Kalman filter for RUL estimation. In this work also, the author establishes that the use of the extended Kalman filter or PF combined with the FPT may be an interesting approach for future research.

Considering all of the above, in this research effort, we propose three methods to improve the efficiency of sampling strategies in the implementation of particle-filtering-based prognostic algorithms. These methods allow working either with deterministic or probabilistic definitions of the failure hazard zone.

As a case study, a hypothetical self-regenerative degradation process is considered for the computation of the ToF-PMF. The performance of the three methods is evaluated through the Jensen-Shannon Divergence and the computation time, in function of the amount of particles used by the particlefiltering-based prognostic algorithm.

This paper is organized as follows: Section 2 describes the main concepts related to ToF estimation, FPT and particlefiltering-based prognostic algorithms, Section 3 describes the proposed methodology, Section 4 introduces the case study, Section 5 shows the simulations and obtained results, and finally, Section 6 presents the conclusions.

\section{Theoretical FrameWork}

\subsection{Time-of-Failure estimation and First-Passage-Time}

Failure prognostic algorithms use long-term predictions to describe the future trend in time of a SoH indicator of a failing system (or subsystem, or component), aiming to estimate its ToF (Figure 1). To compute these long-term predictions, failure prognostic algorithms should require a complete understanding of the underlying dynamics of the SoH indicator, as well as a proper characterization of the related uncertainty sources, and also a future usage profiles of the system (Diaz et al., 2020). Thus, it is more accurate to state that failure prognostic algorithms allow estimating the ToF-PMF of a failing system.

Generally, the SoH indicator is directly related to a degradation process or a deterioration phenomenon (e.g., erosion, corrosion, or cracking) (Deng, Barros, \& Grall, 2016). This entails that monotonic assumptions may be usually made over the $\mathrm{SoH}$ indicator, because most $\mathrm{SoH}$ degradation/deterioration processes exhibit this kind of behavior (Park \& Bae, 2010).

According to this, the system under observation would incur into a catastrophic failure condition only once, moreover, this 


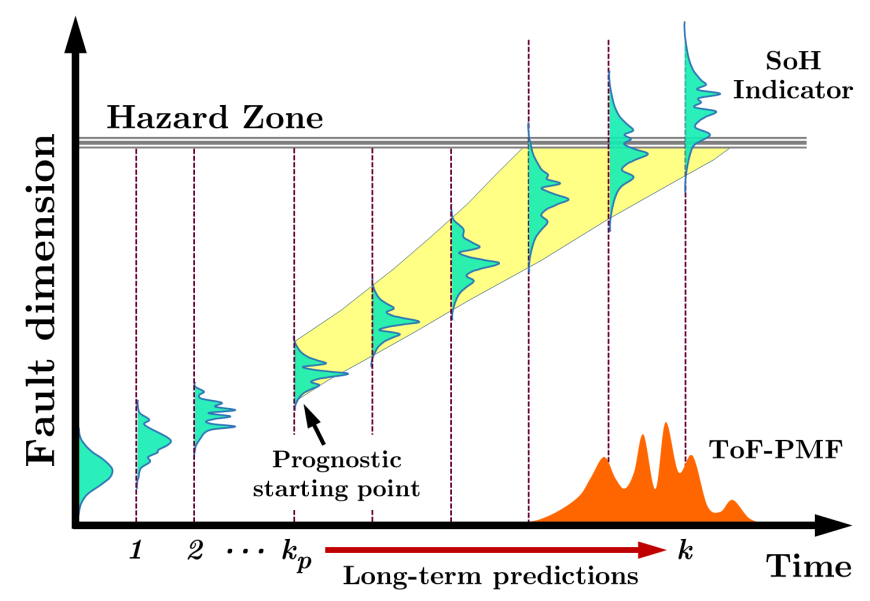

Figure 1. Long-term predictions generated by failure prognostic algorithms.

event could be characterized by a situation where the SoH indicator crosses a predetermined threshold (Zhang, Si, \& $\mathrm{Hu}, 2015)$. For this case, the concept of threshold represents a failure condition defined by collected data from historical failures of the system. In most cases, the threshold is characterized by using a deterministic value; however, if there is enough historical failure data, it is also possible to characterize the probability of failure events by a likelihood function (Orchard \& Vachtsevanos, 2009). The latter is also called hazard zone, and it is the general way to define a failure condition in PHM. Taking this into account, we can observe that a deterministic threshold corresponds to the simplest hazard zone that can be defined (Acuña \& Orchard, 2018).

In cases where the catastrophic failure condition is assumed to occur only once and where the hazard zone is represented by a deterministic threshold $T$, the ToF can be estimated using the concept of FPT (Deng et al., 2016; Jaskowski \& van Dijk, 2015). Mathematically speaking, let $\left\{x_{k}, k \geq 0\right\}$, with $x \in \mathbb{R}, k \in \mathbb{N} \cup\{0\}$, be a scalar discrete-time stochastic process that characterizes the evolution in time of the $\mathrm{SoH}$ indicator, and where it is assumed that the degradation condition monotonically increases its severity in time. Then, the ToF of the stochastic process $x_{k}$ at time $k$, given that we have acquired measurements $y \in \mathbb{R}$ until time $k_{p}$, is computed according to Eq. (1):

$$
\operatorname{ToF}\left(k_{p}\right)=\inf \left\{k:\left\{k>k_{p}\right\} \wedge\left\{x_{k} \geq T \mid y_{1: k_{p}}\right\}\right\}
$$

It is important to mention that, for simplicity in the notation, we consider $\operatorname{ToF}=\operatorname{ToF}\left(k_{p}\right)$ in upcoming equations.

With the definition presented in Eq. (1) and the Law of Total Probability, the authors in Jaskowski and van Dijk (2015) obtained a recursive way to compute the ToF-PMF, $\mathcal{P}(T o F=k)$, given by Eq. (4), as from Eq. (2) and considering $\mathcal{P}\left(x_{k} \geq T \mid T_{o F}=k\right)=1$ in Eq. (3):

$$
\begin{gathered}
\mathcal{P}\left(x_{k} \geq T\right)=\sum_{j=k_{p}+1}^{k} \mathcal{P}\left(x_{k} \geq T \mid \text { ToF }=j\right) \mathcal{P}(\text { ToF }=j) \\
\mathcal{P}\left(x_{k} \geq T\right)=\mathcal{P}\left(x_{k} \geq T \mid \text { ToF }=k\right) \mathcal{P}(\text { ToF }=k)+ \\
\sum_{j=k_{p}+1}^{k-1} \mathcal{P}\left(x_{k} \geq T \mid \text { ToF }=j\right) \\
\mathcal{P}(\text { ToF }=k)=\mathcal{P}\left(x_{k} \geq T\right)-\sum_{j=k_{p}+1}^{k-1} \mathcal{P}\left(x_{k} \geq T \mid \text { ToF }=j\right)
\end{gathered}
$$

As stated above, the expression in Eq. 4 allows computing the ToF-PMF considering a deterministic threshold; besides, in some particular cases for the $\mathrm{SoH}$ indicator random process, an exact and closed-form of the ToF-PMF can be obtained ( $\mathrm{Si}$, Wang, Chen, Hu, \& Zhou, 2013). However, if a probabilistic threshold is considered as the failure condition, the recursion in Eq. (3) is no longer valid, and there is no closed-form solution to the problem of computing the PMF-ToF of the faulty system.

\subsection{Particle Filter based Prognostics algorithms for Time-of-Failure estimation}

In PHM, there are different failure prognostic algorithms proposed to address the prognostic problem; however, many researchers have preferred probability-based methods. This is owing to the possibility to include the notion of uncertainty. A widely used failure prognostic algorithm is based on the PF, which is suitable for both on-line learning systems and state estimation of uncertain systems (Arulampalam, Maskell, Gordon, \& Clapp, 2002). In this sense, particlefiltering-based prognostic algorithms aim at approximating the $\mathrm{SoH}$ indicator PDF by a set of weighted samples (called "particles"). Authors in Orchard and Vachtsevanos (2009) define a theoretical framework and provide the necessary procedures with the purpose of estimating the ToF-PMF, as well as obtaining a proper characterization of it in accordance with Eq. (5):

$$
\mathcal{P}(T o F=k)=\sum_{i=1}^{N_{p}} w_{k}^{(i)} \mathcal{P}\left(x_{k}^{(i)} \geq T \mid y_{1: k_{p}}\right)
$$

where $w_{k}^{(i)} \mathcal{P}\left(x_{k}^{(i)} \geq T \mid y_{1: k_{p}}\right)$ is the probability of being in the event of catastrophic failure, conditional on a specific particle, and $\left\{w_{k}^{(i)}\right\}_{i=1}^{N_{p}}$ are the particle weights at time $k$.

As the expression in Eq. (4), the Eq. 5 is also valid for a system that will reach the condition of catastrophic failure only once given a deterministic threshold. It is noteworthy to highlight these two elements because, in the case of using a probabilistic threshold, the mathematical expression in Eq. (5) is not valid. Otherwise, in case of the $\mathrm{SoH}$ indicator represents a regenerative system, the particles of the particle-filtering- 
based prognostic algorithm could cross the threshold several times, which could lead to a mathematically erroneous estimation of the ToF-PMF (the sum of all the probabilities of the PMF higher than 1). Due to this, in this work, we propose three methods inspired in the concept of FPT, which are capable of obtaining, in an efficient way, an empirical approximation of the ToF-PMF for particle-filtering-based prognostic algorithms. These methods can be used for both nonregenerative and regenerative systems, as well as any kind of hazard zone.

\subsection{The Jensen-Shannon Divergence}

Divergence is a mathematical concept used in different research topics with the purpose of measuring the dissimilarity between PMFs. An example of this is the KullbackLeibler (KL) Divergence. However, the KL-Divergence is not a true metric for the dissimilarity between PMFs because it does not comply with the symmetry property. Instead, we use the Jensen-Shannon (JS) Divergence, which is based on KL-Divergence. Indeed, from a mathematical point of view (Osán, Bussandri, \& Lamberti, 2018), given two random discrete distributions $P=\left\{p_{1}, p_{2}, \ldots, p_{n}\right\}$ and $Q=\left\{q_{1}, q_{2}, \ldots, q_{n}\right\}$ the KL-Divergence is defined by:

$$
D_{K L}(P, Q)=\sum_{i=1}^{n} p_{i} \log \left(\frac{p_{i}}{q_{i}}\right)
$$

and the symmetric JS-Divergence is defined as:

$$
D_{J S}(P, Q)=\frac{1}{2}\left[D_{K L}\left(P, \frac{P+Q}{2}\right)+D_{K L}\left(Q, \frac{P+Q}{2}\right)\right] .
$$

\section{TIME-OF-FAILURE ESTIMATION METHOdS FOR Particle Filter-Based Prognostics algo- RITHMS}

As stated above, particle-filtering-based prognostic algorithms approximate the PDF of the SoH indicator by a set of particles in each instant time in the absence of measurements and allow computing the ToF-PMF when the particles reach (or cross) the predefined hazard zone. These so called hazard zones can be classified in two types according on how the threshold is defined. The first type uses a deterministic threshold, while the other type uses a probabilistic threshold through a failure likelihood function. For both approaches, the ToF computation will depend on the kind of hazard zone considered. In other words, when a deterministic threshold is considered, the ToF for each particle, given that we have acquired measurements until time $k_{p}$, is computed according to Eq. (8):

$$
T o F^{(i)}=\inf \left\{k:\left\{k>k_{p}\right\} \wedge\left\{x_{k}^{(i)} \geq T \mid y_{1: k_{p}}\right\}\right\} .
$$

Otherwise, when a probabilistic hazard zone is considered, the ToF for each particle is computed by means of Eq. (9):

$$
T_{o} F^{(i)}=\inf \left\{k:\left\{k>k_{p}\right\} \wedge\left\{F\left(x_{k}^{(i)}\right)=1\right\}\right\},
$$

where $F(\cdot)$ is the failure likelihood function, that denotes the failure condition of the given particle, which is defined by:

$$
F\left(x_{k}^{(i)}\right)=\left\{\begin{array}{cc}
1 & \text { failure condition } \\
0 & \text { operative condition }
\end{array},\right.
$$

$F(\cdot)$ can be understood as a realization of a Bernoulli process, where the probability of the event is a function of the failure likelihood function that defines the hazard zone and the position of each particle $x_{k}^{(i)}$ in the state space, i.e. $F\left(x_{k}^{(i)}\right) \sim \operatorname{Bernoulli}\left(p\left(x_{k}^{(i)}\right)\right)$. In this case, $p$ is a function that denotes the nonlinear mapping $\mathbb{R} \rightarrow[0,1]$ according to:

$$
p(x)=\left\{\begin{array}{cc}
1 & x \geq T^{+} \\
p * & x \in\left(T^{-}, T^{+}\right) \\
0 & x \leq T^{-}
\end{array}\right.
$$

where $T^{-}$and $T^{+}$indicate the lower and upper bounds of the hazard zone.

On account of the ToF computation previously exposed, the ToF-PMF can be computed through the counting of the particles (and the summation of their respective weights) that have reached the failure condition for the first time.

Considering all the prior concepts, we propose three methods inspired in particle-filtering-based prognostic algorithms, and the FPT concept, to compute the ToF-PMF. Each method differs from the others in the treatment of the particles and their weights. For example, each of these methods consider the weights of the particles as follows:

- Method 1: All the particles have the same weight.

- Method 2: The particles have different weights.

- Method 3: The weights of the particles are re-computed depending on the number of particles that enter into the failure condition.

Furthermore, it is noteworthy to recall that the proposed methods are based also in the concept of FPT, therefore, the three methods only count the particles that enter into the failure condition for the first time. This includes the cases where regenerative systems are prognosticated, since the particles may reach the failure condition more than one time. In this case, the failure condition is considered using one of the following criteria: i) when the particles cross the threshold $T$ if it was a deterministic hazard zone, or ii) when $F\left(x_{k}^{(i)}\right)=1$ for a probabilistic hazard zones. 


\subsection{First Method}

The first method considers the scenario in which all the particles have the same weight when long-term prediction are performed by the particle-filtering-based prognostic algorithm (e.g., when a resampling step is applied before starting the long-term predictions). With this in mind, the ToF-PMF computation will only consider the first time instant in which the particles reach the failure condition.

An illustration of the main idea in which this method is based is shown in Figure 2. Moreover, the procedure to compute the ToF-PMF is detailed in Algorithm 1 for a deterministic hazard zone, and in Algorithm 2, for a probabilistic hazard zone. It is important to specify that, for all proposed algorithms, we use the term Prediction_horizon to denote the time period in which long-term predictions are computed, whereas the Prediction_step corresponds to a predictive update of the state vector that is computed using the process model.

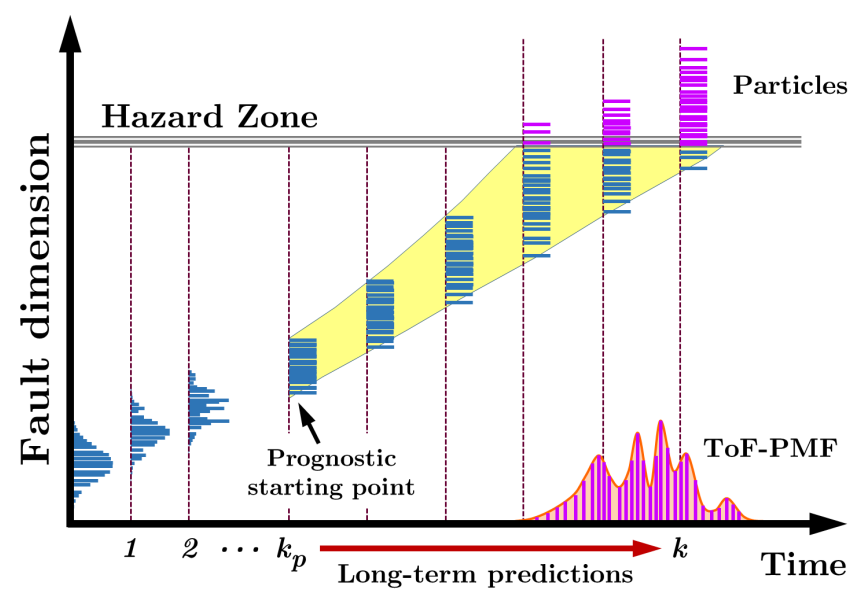

Figure 2. Computation of ToF-PMF using the first method. Every time a blue particle enters the hazard zone, its failure status is considered to form the ToF-PMF, then discarded (magenta particles). The weight of the particles is illustrated with a line, every line has the same weight.

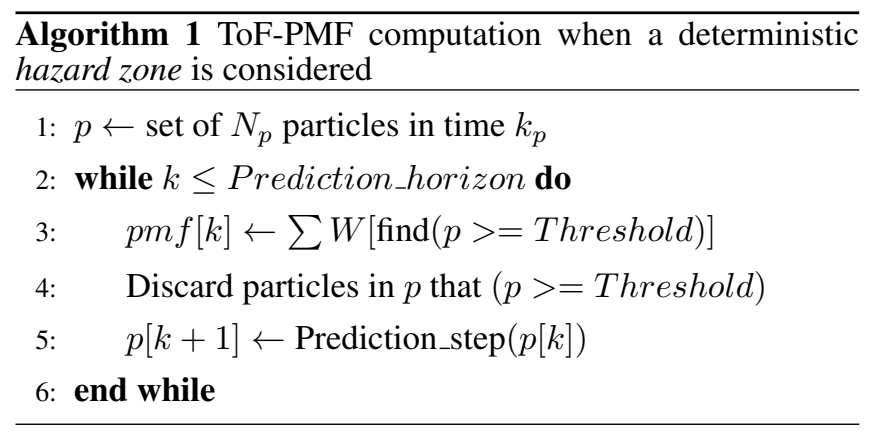

\subsection{Second Method}

The second method is similar to the first method. The only difference is that Method 2 considers that the particles

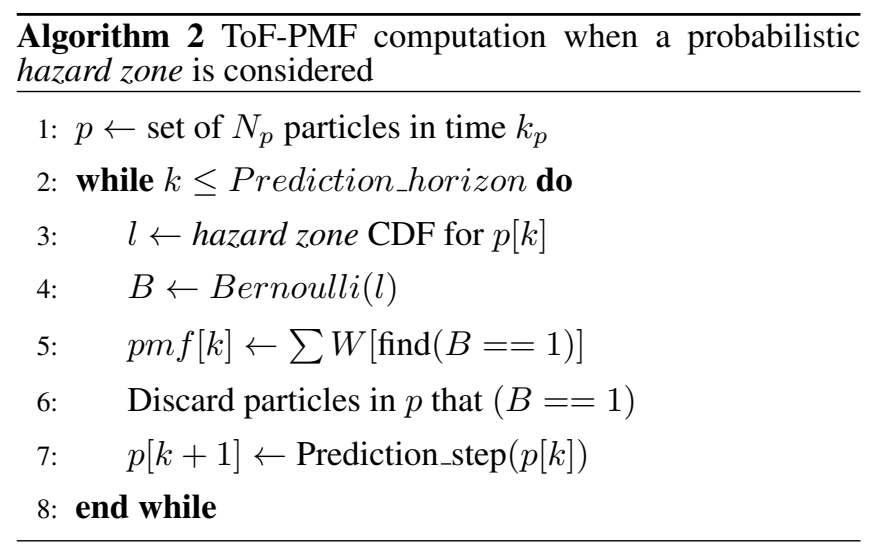

have different weights when long-term prediction are performed by the particle-filtering-based prognostic algorithm (e.g., when the resampling step is not applied before starting the long-term predictions). An example of this method is shown in Figure 3. The general procedure to compute the ToF-PMF through this method is also detailed by Algorithms 1 and 2.

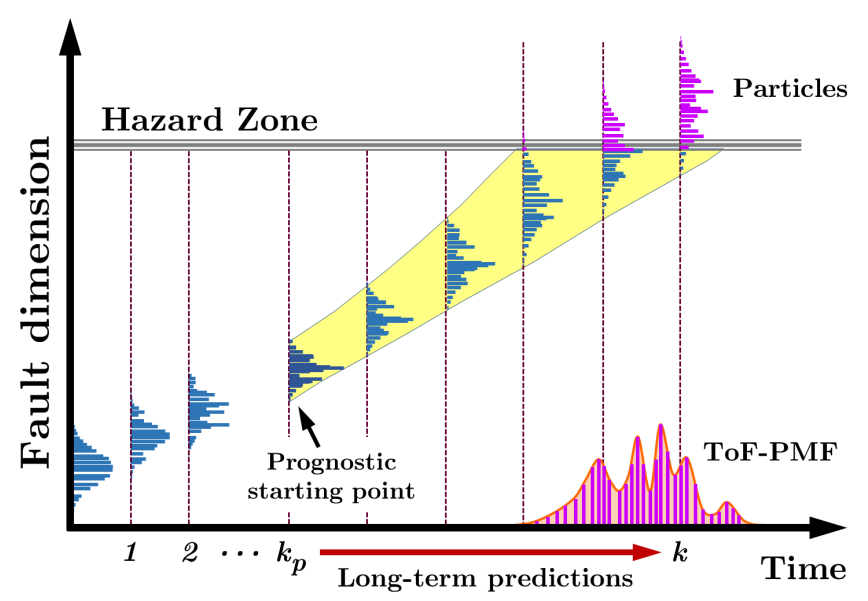

Figure 3. Computation of ToF-PMF using the second method. As the first method the blue particles are used to form the PMF, now their weights are not uniform, they are sampled from a normal distribution from $N(E(k), \sigma(k))$.

\subsection{Third Method}

The third method is similar to Method 1 , in the way that, the long term predictions are performed using particles with the same weight, and considering the first time instant in which the particles reach the failure condition. However, this method is proposed to improve the ToF-PMF characterization by increasing the number of particles.

In this method, at every time instant that a particle enters the failure condition ( $p \geq T$ or $F(p)=1$ ), a new particle is inserted to the "healthy particles" $\left(H_{p}\right)$ set by using the multi- 
nomial resampling approach (Douc \& Cappe, 2005). With the addition of this new particle, the weights of the $H_{p}$ set are recalculated, with the aim of maintaining all these particles with the same weight, and also considering that the sum of the whole set of particles must be equal to 1 .

Figure 4 illustrates the proposed methodology. The $H_{p}$ set is represented with blue. As time passes, some particles enter into the hazard zone. These particles are colored in magenta, and they are replaced with new particles, colored with red. Once the substitution is made and the weights of the $H_{p}$ set is recalculated, the algorithm continues.

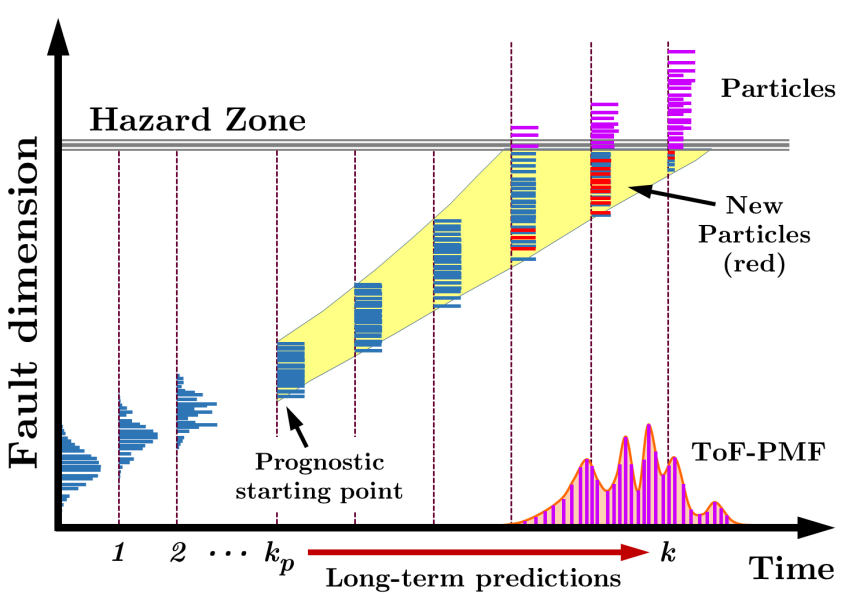

Figure 4. Computation of ToF-PMF using the third method. Here the weights are changing according to the particles that are resampled. The weight of the remaining particles is distributed to the blue and red particles to maintain the integral of the ToF-PMF equal to one.

The procedure to compute the ToF-PMF by Method 3 is detailed in Algorithm 3 for a deterministic hazard zone, and in Algorithm 4, for a probabilistic hazard zone.

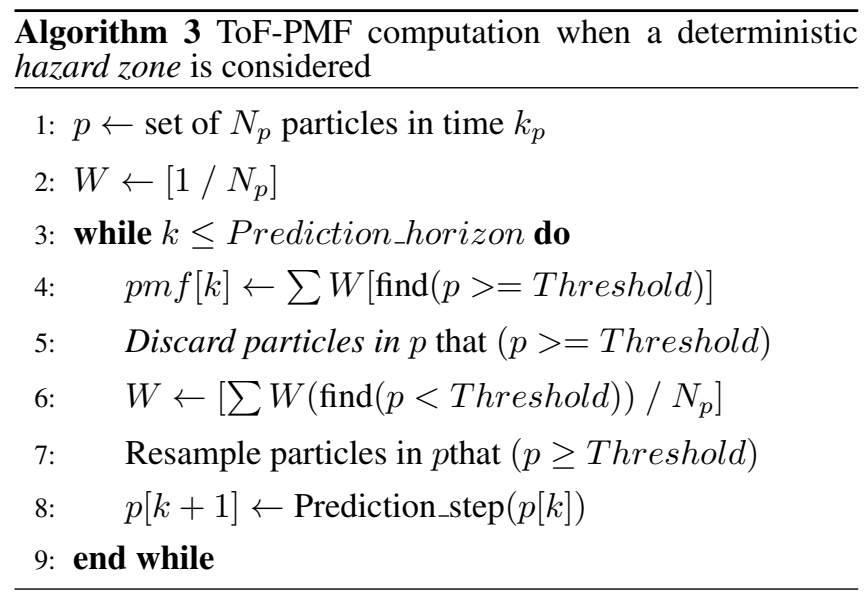

$\overline{\text { Algorithm } 4 \text { ToF-PMF computation when a probabilistic }}$ hazard zone is considered

$$
\begin{array}{ll}
\text { 1: } & p \leftarrow \text { set of } N_{p} \text { particles in time } k_{p} \\
\text { 2: } & W \leftarrow\left[1 / N_{p}\right] \\
\text { 3: } & \text { while } k \leq \text { Prediction_horizon do } \\
\text { 4: } & p \leftarrow \text { Prediction_step }(p) \\
\text { 5: } & l \leftarrow \text { hazard zone } \text { CDF for } p[k] \\
\text { 6: } & B \leftarrow \text { Bernoulli }(l) \\
\text { 7: } & p m f[k] \leftarrow \sum W[\text { find }(B==1)] \\
\text { 8: } & \text { Discard particles in } p \text { that }(B==1) \\
\text { 9: } & W \leftarrow\left[\sum W(\text { find }(B==1)) / N_{p}\right] \\
\text { 10: } & \text { Resample particles in } p \text { that }(B==1) \\
\text { 11: } & \text { end while }
\end{array}
$$

\section{Case Study}

A hypothetical self-regenerative system was considered as a case study to analyze the proposed methodology. This hypothetical degradation process was designed to represent a strong self-regenerative phenomenon with the aim to evaluate the proposed methods in a challenging scenario. It is also important to note that the self-regenerative model is only used to generate the long-term predictions of the state variable, which means that the proposed case study represents only the prognosis stage of a PHM application. Therefore, any previous estimation stage is assumed as correct, and the initial state $(x(0))$ for the proposed self-regenerative model corresponds to the prognostic starting point of a particle-filter-based prognostics algorithm (i.e., $k_{p}$ in Figures 1, 2, 3, and 4).

The self-regenerative system is modeled by Eq. 12, with $x(0)=1$. Model parameters $a$ and $b$ are set to 1.06 and 0.935 , respectively. In addition, the process noise is modeled as a Gaussian distribution, $\omega_{k} \sim \mathcal{N}(0,6.4 e-3)$, and the prediction horizon $H$ equal to 156 . The hazard zone is characterized with the threshold value $T=20$ in the deterministic case, and the Gaussian distribution $\mathcal{N}(20,0.5)$ in a probabilistic scenario.

$$
x_{k+1}= \begin{cases}a \cdot x_{k}+\omega_{k} & \text { if } k<H / 3 \\ a \cdot b \cdot x_{k}+\omega_{t} & \text { if } H / 3 \leq k<2 H / 3 \\ a \cdot x_{k}+\omega_{k} & \text { if } 2 H / 3 \leq k \leq H\end{cases}
$$

To compute the ground truth ToF-PMF for the proposed case study, Monte Carlo (MC) simulations were performed. With the purpose of obtain an acceptable representation of the ground truth ToF-PMF, one million of MC simulations were considered. In Figure 5, an example of the MC simulations for the regenerative system is shown. Figure 6 shows the corresponding ground truth ToF-PMF for the both kinds of hazard zones. 


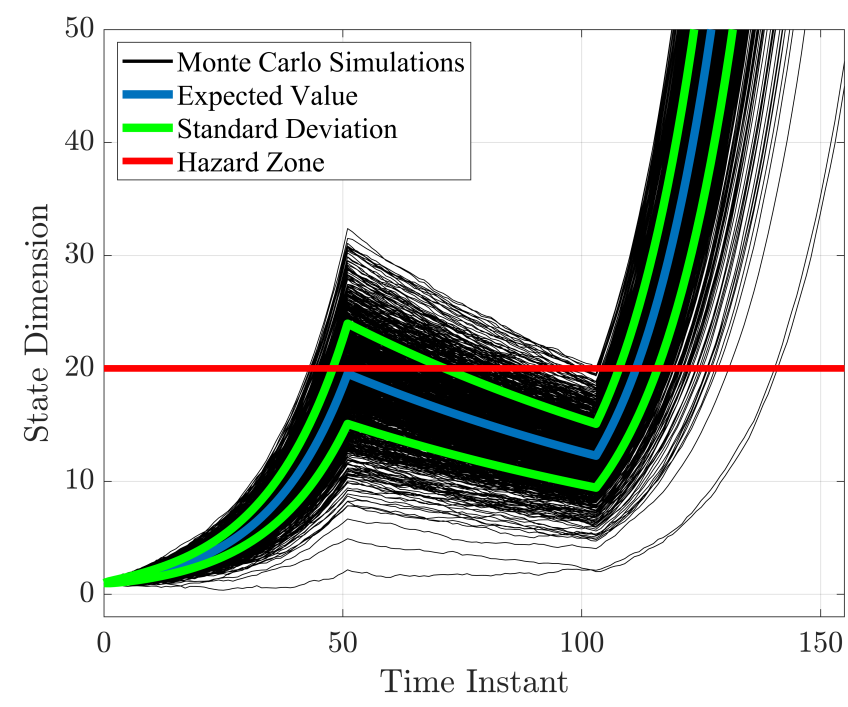

Figure 5. Monte Carlo simulations for the hypothetical case study (self-regenerative system).
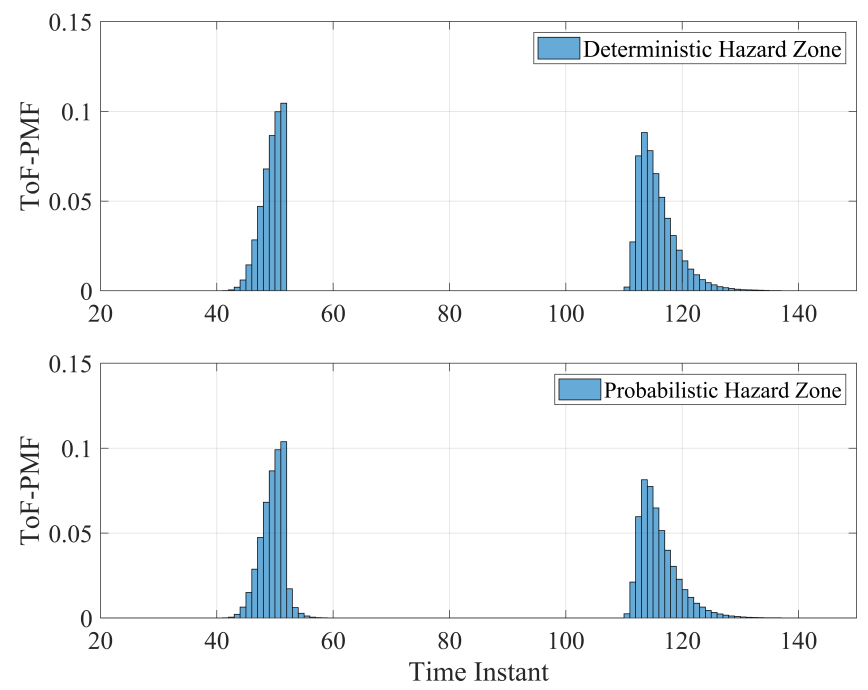

Figure 6. Ground Truth ToF-PMF for a deterministic hazard zone (upper plot) and a probabilistic hazard zone (lower plot).

\section{RESULTS}

To evaluate the ToF-PMF characterization of the proposed methods and their efficiency, the three algorithms were tested using the self-regenerative process presented in the case study. The ToF-PMF estimation obtained by the three methods were compared to the ground truth ToF-PMF obtained in the case study. The software used for the simulations was Matlab R2017, and the hardware corresponds to an Intel ${ }^{\circledR}$ Core $^{\mathrm{TM}}$ i5-8250U CPU @ 1.60GHz 1.80GHz, and $12 \mathrm{~GB}$ of RAM.

The ToF-PMF characterization was evaluated both through the JS Divergence (Osán et al., 2018), and the execution time.
In both cases, they were represented as a function of the amount of particles $\left(N_{p}\right)$ used by the particle-filtering-based prognostic algorithm. The amount of particles were varied from 100 to 500, and increased by hundreds. Additionally, one hundred iterations per each of the $N_{p}$ defined were considered to carry out an statistical analysis.

Finally, the ToF-PMF estimation for the case study considered the two kinds of hazard zones described above: deterministic threshold and failure likelihood function. In this way, the results are organized according to the kind of hazard zones, as stated below.

\subsection{Deterministic hazard zone}

The results for the three methods, when a deterministic threshold is considered are shown in Figure 7. The upper plot compares the results obtained for the JS-Divergence as a function of the amount of particles, while the lower plot compares the execution times for the three methods.
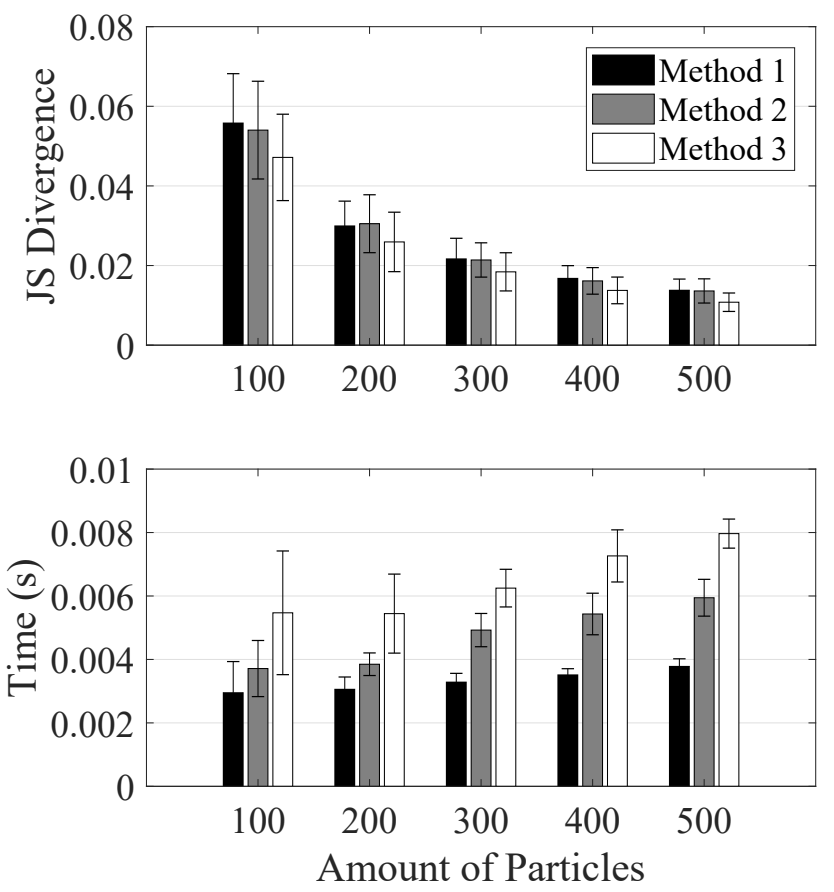

Figure 7. Comparison between the three methods over a deterministic hazard zone. JS-Divergence is used to compare the level of approximation of the ToF-PMF and the time of computation is measured. Each plot shows the mean and standard deviation of each method.

For a given amount of particles, the best characterization of the ToF-PMF is obtained using Method 3, as seen in upper plot of Figure 7, followed by Method 2 and 1. This can be explained by the formulation of Method 3 which has the effect of maintaining a constant amount of particles available to compute the ToF-PMF in every time instant. However, this 
method considers a resampling algorithm that requires major computation efforts to compute the ToF-PMF in comparison with Method 1 and 2. This is illustrated in the execution time plot of Figure 7, where Method 1 and 2 are in ascending order, since the complexity of them is also increased, and the one with major complexity is the slowest of the three proposed methods.

According to Figure 7 and the obtained results regarding the JS-Divergence the performance of the three methods present a considerable deviation with a few amount of particles. But that trend tends to decrease with the increasing amount of particles considered to compute the ToF-PMF with each method. Furthermore, the execution time does increase for the three methods when the amount of particles is also increased.

Finally, Figure 8 shows the comparison between the ToFPMF obtained by each method using 200 particles, and the ground truth ToF-PMF.
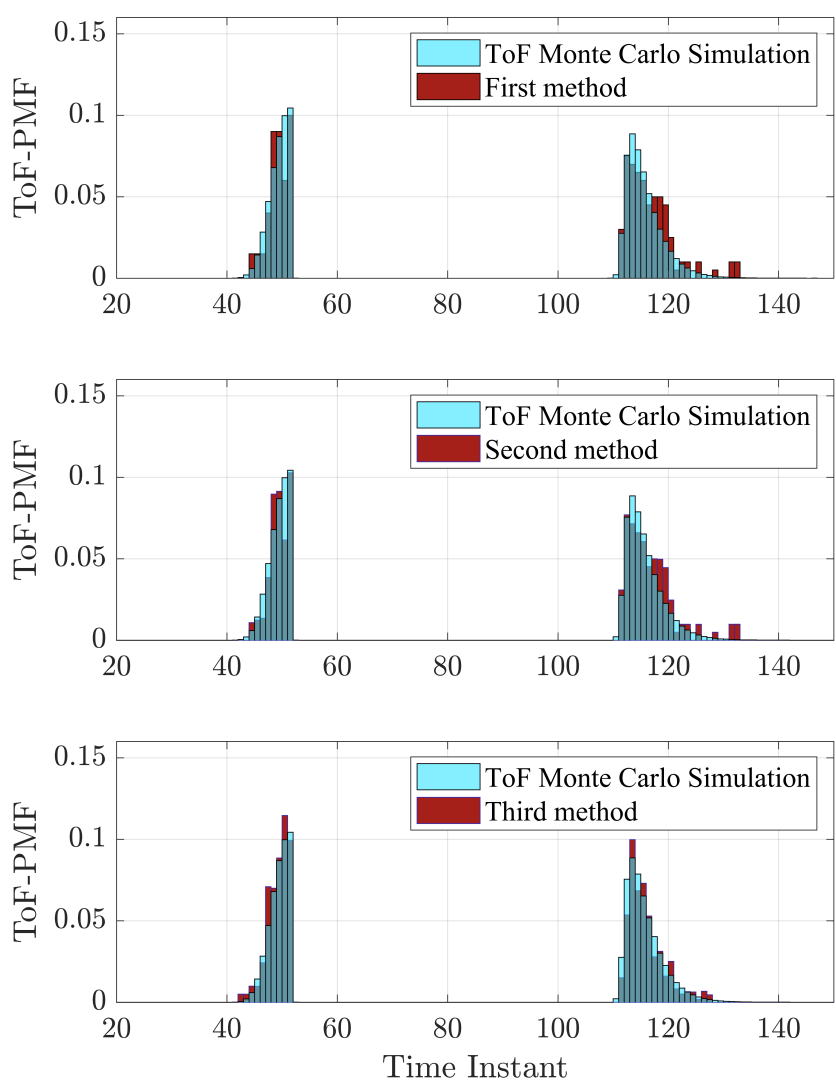

Figure 8. Comparison of the PMFs obtained by the three proposed methods. The corresponding JS-Divergence for Method 1, 2, and 3 are equal to $0.03457,0.03321$, and 0.01592 , respectively.

\subsection{Probabilistic hazard zone}

When the hazard zone is characterized by a failure likelihood function, the three methods behave similar to the determinis- tic case. In Figure 9, in the upper plot the JS-Divergence for the three methods shows that the Method 3 is the one that better characterizes the ToF-PMF of the ground truth, and the Method 1 and Method 2 behave in the same manner as for the deterministic threshold.
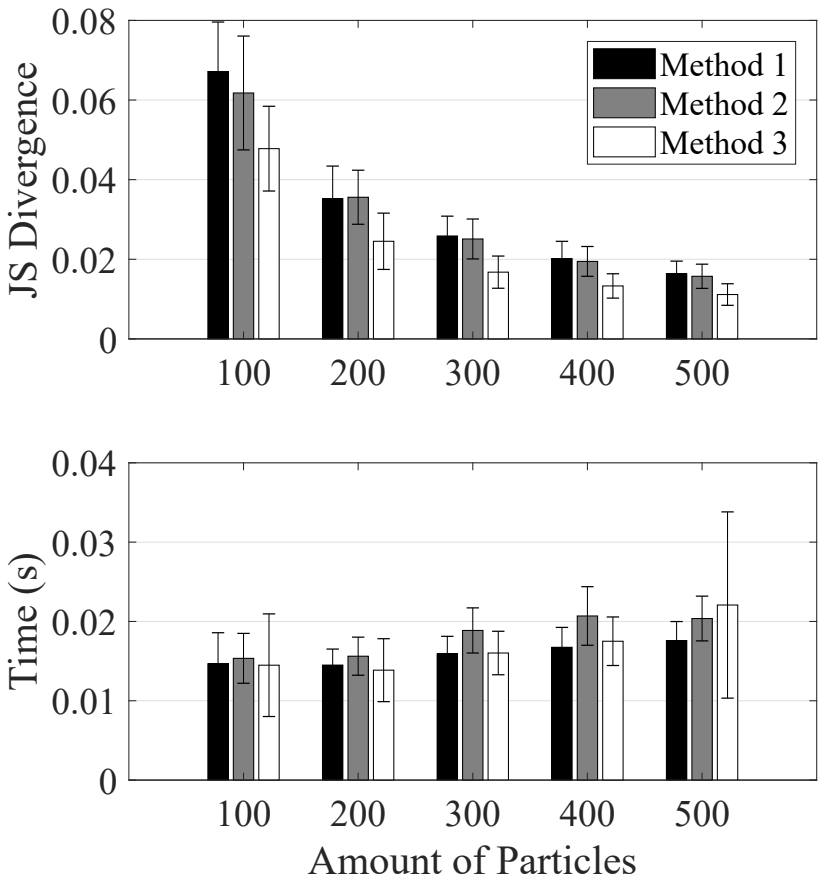

Figure 9. Comparison between the three methods over a deterministic hazard zone. JS-Divergence is used to compare the level of approximation of the ToF-PMF and the time of computation is measured. Each plot shows the mean and standard deviation of the respective measure per method.

Moreover, when the execution time is compared all three methods are less efficient in time when the number of particles is increased. This can be noted in the lower plot of Figure 9. This behavior can be explained by the implementation of the Bernoulli process in the computation of the failure status of the particles, since this new step takes almost 70, 60 and 40 percent of the total execution time per method, respectively. Also, the dispersion does not show a trend for any of the proposed methods, but for Method 3 a greater dispersion in execution time is measured, and presents an independent behavior respect to the amount of particles.

Figure 10 shows a comparison of the resulting PMF of the three methods with a hazard zone characterized by a failure likelihood function. This example considers the case of 200 particles, and it is contrasted with the Monte Carlo simulations of the Case Study.

Finally, it is important to mention that simulations with less amount of particles were performed, although the observed behavior was similar to the one exposed in this article. 

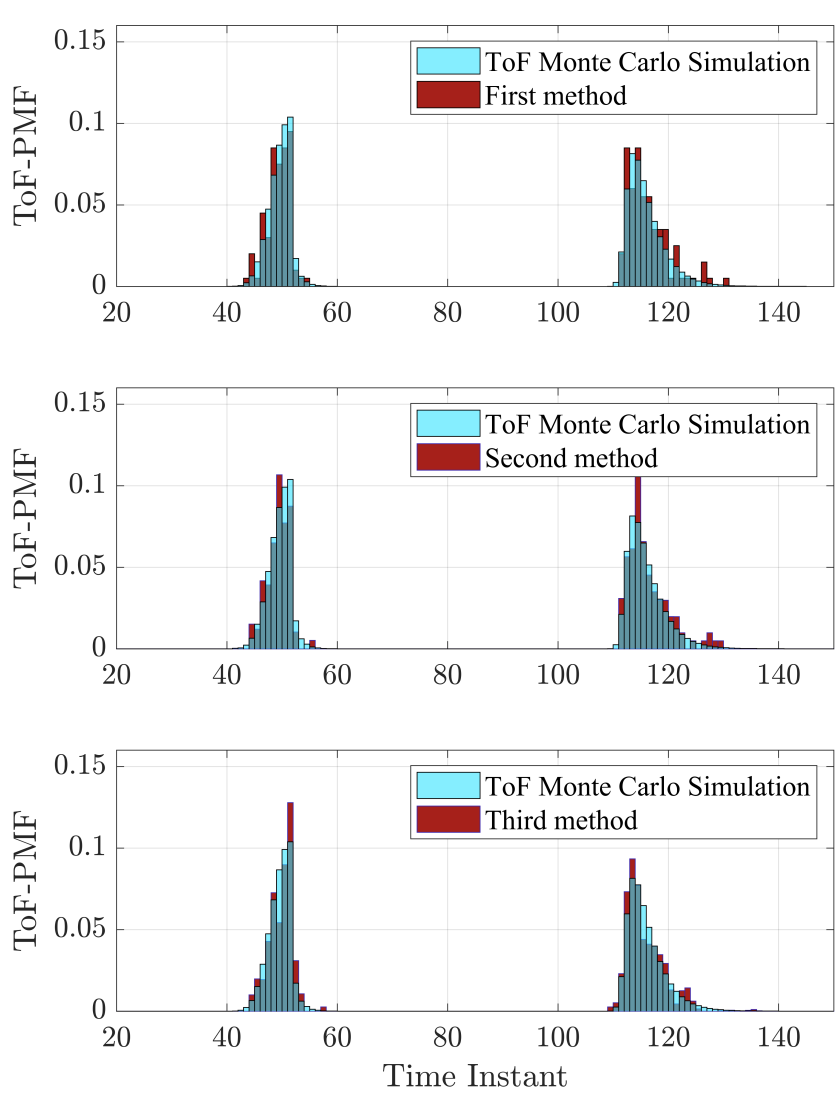

Figure 10. Comparison of the PMFs obtained by the three proposed methods. The corresponding JS-Divergence for Method 1, 2, and 3 are equal to $0.03115,0.02968$, and 0.02246 , respectively.

\section{Conclusions}

In this paper three methods were proposed to compute the ToF-PMF based on FPT, and considering two kinds of hazard zones. The three proposed methods were evaluated using a hypothetical self-regenerative degradation process.

The three methods were capable of approximating the ToFPMF, compared to the ground truth ToF-PMF, at a reasonable level, and in an efficient manner. Therefore, the three proposed methods may be used in particle-filtering-based prognostic implementations for different kinds of processes and hazard zones.

When the results were focused on the efficiency of the execution time, they showed that the proposed methods can be implemented in real conditions by particle-filtering-based prognostic algorithms. However, the trade-off between ToF-PMF approximation and execution times must be always considered.

For future work, we propose to test this methodology considering real data, to compare its performance with stateof-the-art methodologies. Also, to evaluate the behavior of each of the algorithms in a context with limited computational power.

\section{ACKNOWLEDGMENT}

The authors would like to acknowledge and sincerely thank Dr. David Acuña for his significant contributions, support, and guidance regarding the theoretical aspects that inspired this research effort. The work of V. Quintero was supported by the National Research System of Panamá (SNISENACYT). The work of A. Perez was supported by the University of Costa Rica under research project 322-C0-702. The work of M. E. Orchard was supported by FONDECYT Grant 1170044 and the Advanced Center for Electrical and Electronic Engineering, AC3E, Basal Project FB0008, ANID.

\section{REFERENCES}

Acuña, D., \& Orchard, M. (2018). A Theoretically Rigorous Approach to Failure Prognosis. In Annual conference of the phm society (p. 14).

Arulampalam, M. S., Maskell, S., Gordon, N., \& Clapp, T. (2002). A tutorial on particle filters for online nonlinear/non-Gaussian Bayesian tracking. IEEE Transactions on Signal Processing, 50(2), 174-188.

Bakshi, G., \& Panayotov, G. (2010). First-passage probability, jump models, and intra-horizon risk. Journal of Financial Economics, 95, 20-40.

Deng, Y., Barros, A., \& Grall, A. (2016). Degradation modeling based on a time-dependent ornstein-uhlenbeck process and residual useful lifetime estimation. IEEE Transactions on Reliability, 65(1), 126-140.

Diaz, C., Quintero, V., Perez, A., Jaramillo, F., BurgosMellado, C., Rozas, H., ... Cardenas, R. (2020). Particle-filtering-based prognostics for the state of maximum power available in lithium-ion batteries at electromobility applications. IEEE Transactions on Vehicular Technology, 69(7), 7187-7200.

Douc, R., \& Cappe, O. (2005). Comparison of resampling schemes for particle filtering. In Ispa 2005. proceedings of the 4th international symposium on image and signal processing and analysis, 2005. (pp. 64-69).

Janssen, J., Manca, O., \& Manca, R. (2013). Applied diffusion processes from engineering to finance. John Wiley $\&$ Sons, Ltd.

Jaskowski, M., \& van Dijk, D. (2015). First-Passage-Time in Discrete Time.

Liu, Y., Zhao, G., \& Peng, X. (2019). Deep learning prognostics for lithium-ion battery based on ensembled long short-term memory networks. IEEE Access, 7, 155130-155142.

McKenzie, H. W., Lewis, M. A., \& Merrill, E. H. (2009). First passage time analysis of animal movement and insights into the functional response. Bulletin of math- 
ematical biology, 71(1), 107-129.

Ng, S. S., Xing, Y., \& Tsui, K. L. (2014). A naive bayes model for robust remaining useful life prediction of lithium-ion battery. Applied Energy, 118, $114-123$.

Orchard, M., Lacalle, M. S., Olivares, B. E., Silva, J. F., Palma-Behnke, R., Estévez, P. A., ... CortésCarmona, M. (2015). Information-theoretic measures and sequential monte carlo methods for detection of regeneration phenomena in the degradation of lithiumion battery cells. IEEE Transactions on Reliability, 64(2), 701-709.

Orchard, M., \& Vachtsevanos, G. (2009). A particle-filtering approach for on-line fault diagnosis and failure prognosis. Transactions of the Institute of Measurement and Control, 31, 221-246.

Osán, T. M., Bussandri, D. G., \& Lamberti, P. W. (2018). Monoparametric family of metrics derived from classical Jensen-Shannon divergence. Physica A: Statistical Mechanics and its Applications, 495, 336-344.

Park, J. I., \& Bae, S. J. (2010). Direct Prediction Methods on Lifetime Distribution of Organic Light-Emitting Diodes From Accelerated Degradation Tests. IEEE Transactions on Reliability, 59(1), 74-90.

Pei, H., Hu, C., Si, X., Zheng, J., Zhang, Q., Zhang, Z., \& Pang, Z. (2019). Remaining useful life prediction for nonlinear degraded equipment with bivariate time scales. IEEE Access, 7, 165166-165180.

Pola, D. A., Navarrete, H. F., Orchard, M. E., Rabié, R. S., Cerda, M. A., Olivares, B. E., ... Pérez, A. (2015). Particle-filtering-based discharge time prognosis for lithium-ion batteries with a statistical characterization of use profiles. IEEE Transactions on Reliability, 64(2), 710-720.

Qu, J., Liu, F., Ma, Y., \& Fan, J. (2019). A neural-networkbased method for rul prediction and soh monitoring of lithium-ion battery. IEEE Access, 7, 87178-87191.

Rozas, H., Jaramillo, F., Perez, A., Jimenez, D., Orchard, M. E., \& Medjaher, K. (2020). A method for the reduction of the computational cost associated with the implementation of particle-filter-based failure prognostic algorithms. Mechanical Systems and Signal Processing, 135, 106421.

Si, X.-S. (2015). An adaptive prognostic approach via nonlinear degradation modeling: Application to battery data. IEEE Transactions on Industrial Electronics, 62(8), 5082-5096.

Si, X.-S., Wang, W., Chen, M.-Y., Hu, C.-H., \& Zhou, D.-H. (2013). A degradation path-dependent approach for remaining useful life estimation with an exact and closedform solution. European Journal of Operational Research, 226(1), 53-66.

Skaf, Z. (2015). Prognostics: Design, implementation, and challenges. Curran Associates.

Wang, C., Lu, N., Cheng, Y., \& Jiang, B. (2019). A data-driven aero-engine degradation prognostic strategy. IEEE transactions on cybernetics, 1-11.

Wei, J., Dong, G., \& Chen, Z. (2018, July). Remaining useful life prediction and state of health diagnosis for lithiumion batteries using particle filter and support vector regression. IEEE Transactions on Industrial Electronics, 65(7), 5634-5643.

Xu, X., Yu, C., Tang, S., Sun, X., Si, X., \& Wu, L. (2019). State-of-health estimation for lithium-ion batteries based on wiener process with modeling the relaxation effect. IEEE Access, 7, 105186-105201.

Zhang, Z., Si, X., \& Hu, C. (2015). An Age- and StateDependent Nonlinear Prognostic Model for Degrading Systems. IEEE Transactions on Reliability, 64(4), 1214-1228.

Zou, Y., Hu, X., Ma, H., \& Li, S. E. (2015). Combined state of charge and state of health estimation over lithiumion battery cell cycle lifespan for electric vehicles. Journal of Power Sources, 273, 793 - 803.

\section{BIOGRAPHIES}

Francisco Jaramillo received the B.Sc. degree in electronics engineering from Universidad de La Frontera, Temuco, Chile and the Doctorate degree in electrical engineering from the University of Chile. Currently, he is a Researcher at the Fault Diagnosis and Failure Prognosis Laboratory with the University of Chile. His research interests include machine learning, control systems, and fault diagnosis and failure prognosis based on Bayesian algorithms with applications to nitrogen removal in pilot-scale sequencing batch reactors for wastewater treatment plants, and structural health monitoring of wind turbine blades.

Martín Valderrama is a B.Sc. student in Electrical Engineering at the University of Chile. Currently he is a Research Assistant at the Fault Diagnosis and Failure Prognosis Laboratory of the University of Chile. His research interests include Fault Prognosis and Health Management for decision making, machine learning and Deep Learning for Image Recognition.

Vanessa Quintero received the B.Sc degree in electronics and telecommunication engineering from the Universidad Tecnológica de Panamá in 2007 and the Doctorate in electrical engineering degree from the University of Chile in 2019. Currently, she is a full-time Assistant Professor at the Faculty of Electrical Engineering at the Universidad Tecnológica de Panamá, and member of the National Research System (SNI) of Panama. Her research interests include estimation, prognostics with applications to batteries and the design of communication protocols.

Aramis Pérez received the B.Sc. degree and Licentiate degree in electrical engineering, and the master degree in business administration in general management major from the University of Costa Rica. He received the Doctorate degree in electrical engineering degree from the University of Chile. Currently, he is a full time Assistant Professor with the School of Electrical Engineering, University of Costa Rica. His research interests include parametric and non-parametric mod- 
eling, system identification, data analysis, and applications to electromobility, energy storage and photovoltaic systems.

Marcos E. Orchard received the M.S. and Ph.D. degrees in electrical and computer engineering from The Georgia Institute of Technology, Atlanta, GA, USA, in 2005 and 2007 respectively. Currently he is a Professor with the Department of Electrical Engineering at Universidad de Chile and Associate Researcher with the Advanced Center for Electrical and Electronic Engineering (UTFSM). He has authored and co-authored more than 100 papers on diverse topics, in- cluding the design and implementation of failure prognostic algorithms, statistical process monitoring and system identification. His research work at the Georgia Institute of Technology was the foundation of novel real-time failure prognosis approaches based on particle filtering algorithms. His current research interests include the study of theoretical aspects related to the implementation of real-time failure prognosis algorithms, with applications to battery management systems, electromobility, mining industry, and finance. Dr. Orchard is a Fellow of the Prognostic and Health Management Society. 